\title{
Enunciación
}

\section{La influencia social de los medios de comunicación en Twitter}

\author{
The Media Social Influence on Twitter
}

\author{
Karines Rodríguez Díaz*, Yamile Haber Guerra*
}

\section{Resumen}

En este artículo se expone cómo se desarrolla la influencia social de los medios de comunicación sobre los usuarios en Twitter, durante la celebración de la VII Cumbre de la Asociación de Estados del Caribe (AEC). La investigación parte del análisis de un corpus de 1500 tuits con las etiquetas temáticas \#CubaCaribe, \#VIICumbreAEC, \#CumbreAEC. Utilizando el análisis del discurso mediado por computador, los resultados muestran la habilidad de influencia de los medios de comunicación a través de patrones de repetición-reacción y en la construcción del consenso sobre la valoración del acontecimiento. El reporte de caso explica cómo los parlamentos mediáticos repercuten en la comunidad de usuarios en el contexto microblogging.

Palabras clave: influencia social, medios de comunicación, educación informal, usuarios, redes sociales.

\begin{abstract}
This report exposes how the media developed social influence in users on Twitter during the VII Summit of the Asociation of Caribbean States (ACS). This research analyses a corpus of 1500 tweets with hashtags \#CubaCaribe, \#VIICumbreAEC, \#CumbreAEC. Using as a method the computer- mediated discourse analysis; the results show skills of institutional media to influence through repetition- reaction standards and the construction of consensus about the assessment of the event. The case report expounds how the media parliaments have repercussions on users community in the microblogging context.
\end{abstract}

Keywords: social influence, media, informal education, users, social network.

\footnotetext{
* Licenciada en Periodismo. Profesora instructora del Departamento de Periodismo de la Universidad de Oriente, Santiago de Cuba, Cuba. Correo electrónico: karines@uo.edu.cu

** Doctora en Ciencias Filológicas. Profesora Titular de la Universidad de Oriente, Santiago de Cuba, Cuba. Correo electrónico: yhaber@uo.edu.cu
} 


\section{Introducción}

Los medios de comunicación son importantes agentes de influencia social, dada su caracterización como fuente oficial de información; de igual forma, son promotores de la educación informal y del establecimiento de valoraciones en la opinión pública. Entender su capacidad para influir sobre su audiencia parte de la naturaleza de la interpretación que los usuarios dan al producto que es la noticia.

La influencia de los medios de comunicación es un tema tratado con anterioridad; desde la teoría de la aguja hipodérmica hasta los estudios de efectos a largo plazo, como agenda setting, gatekeeping o news making, se ocuparon de dar una explicación sobre el poder de los medios y su influencia en la reacción de sus consumidores. La semiótica social, como relación de los medios y las audiencias en la construcción de los signos de la sociedad, es una de las líneas investigativas que, dentro de la comunicación, revisa la organización, distribución, interacción y creación del significado. Autores como Jensen (1997) se preocuparon por la construcción del significado en comunidades interpretativas, con lo que se demostró que el receptor tenía un papel activo en la asimilación de los parlamentos mediáticos. Sin embargo, las teorías antes citadas se produjeron en correspondencia con medios de naturaleza analógica.

Con la evolución de la web, el análisis medio-usuario de internet se ha dirigido a la naturaleza de la plataforma, fundamentalmente a la revisión de la influencia de los medios y los líderes de opinión en situaciones electorales o políticas; por ejemplo, Zappavigna (2011) aplica la lingüística sistémica funcional para determinar la consideración que dieron los usuarios a la figura de Barack Obama en su primera campaña política en Twitter. Autores como Gruzd, Wellman y Takhteyev (2011) analizan el comportamiento asociado a las comunidades virtuales que se crean en el microblogging. Estos investigadores facilitan herramientas metodológicas para entender los patrones de conducta de usuarios en el contexto de comunidades virtuales.

Sin embargo, una de las teorías más cercanas a la determinación de los públicos en la distribución de contenido informativo en la web 2.0 es el gatewatching ${ }^{1}$, tomando como referencia a Burns y Highfield (2015). Estudios del Departamento de Periodismo de la Universidad de Oriente (véase Loo et al., 2015) enfocan la importancia del usuario como agente en la distribución y producción de significados sobre temáticas noticiosas, siguiendo el enfoque teórico ofrecido por Burns y Highfield (2015). No obstante, es necesario realizar investigaciones que aborden las interacciones comunicativas que enfocan el proceso en su conjunto, para, desde el lenguaje, demostrar la influencia de los medios sobre los usuarios en redes sociales, donde la acción de estos últimos es activa. Es esencial probar la efectividad de los medios en casos particulares, sin dejar de lado ninguno de los actores o componentes de los modelos clásicos de comunicación.

El estudio de la producción de significados y socialización en torno a las agendas de los medios de comunicación permite determinar cambios en las dinámicas mediáticas en este entorno comunicativo (Twitter), a partir de un análisis de corpus lingüístico. En el presente artículo se analiza la influencia de los medios de comunicación sobre otros usuarios en la producción de contenidos asociados a la VII Cumbre de la Asociación de Estados del Caribe, celebrada en Cuba, del 2 al 4 de junio de 2016.

Problema de investigación: ¿Cómo se desarrolla la influencia social de los medios de comunicación sobre los usuarios en Twitter?

El objetivo fue explicar las formas en las que se produce la influencia social de los medios de comunicación sobre los usuarios en Twitter, en el caso particular de la VII Cumbre de Asociación de Estados del Caribe.

Para dar respuesta al problema de investigación es preciso entender al medio de comunicación como una cuenta institucional que lo representa en el

1 El gatewatching es la selección de noticias que publican usuarios comunes en la red reproduciendo las fuentes informativas, lo que obliga a los medios de comunicación tradicionales a competir por la atención de los lectores. Es un filtrado de noticias que alcanza su trasmisión a través de enlaces sociales en comunidad web. Se trata de un nuevo tipo de gatekeeping (Canavilhas, 2011) desarrollado por los medios de comunicación social en sus canales, pero también por ciudadanos -los gatewatchers- alrededor de los cuales se construyen nuevas comunidades informales. 
microblogging, si bien la institución mediática no está aislada, sino que forma parte de una comunidad virtual o colectivo de usuarios cuyos mensajes se agrupan a partir de estructuras de organización textual conocidas como hashtags (etiquetas).

Para la consecución del proyecto fue necesario dimensionar las categorías conceptuales que se presentan a continuación.

\section{Referentes conceptuales}

\section{La educación informal de los medios de comunicación}

Si entendemos la educación informal como "aquellos procesos educativos que tienen lugar en el transcurso normal de las relaciones sociales, de la vida cotidiana, y en los que las personas, de manera no organizada, asistemática y con frecuencia no intencional, adquieren y acumulan conocimientos, habilidades, actitudes y modos de discernimiento" (Liceras, 2005, p. 2), comprendemos que su construcción depende, entre otros factores, de la influencia social de los medios de comunicación sobre sus receptores. Dicha influencia comienza como un proceso conceptual y contextual hasta llegar a promover acciones valorativas.

Esa capacidad de influencia se ampara en que estos sujetos están construyendo sus esquemas cognitivos para interpretar la realidad. Los medios construyen la percepción del mundo, son mediadores que potencian una influencia imitativa, que obtienen-poseen un prestigio social (lo que aumenta su credibilidad), con mensajes que se manifiestan a través de varios códigos simultáneos y de recursos tecnológicos (Liceras, 2005).

Sin embargo, existen límites a la capacidad de influencia de los medios. Según Buckingham (2005), los receptores no son pasivos frente a los mensajes de los medios de comunicación, ningún medio tiene efecto ilimitado sobre los receptores. Desde la teoría de los usos y gratificaciones, podemos observar un acercamiento a las condiciones interpersonales que marcan los efectos mediáticos. Las necesidades de los individuos determinan el uso de los medios, y la interpretación, disfrute y adaptación del mensaje están condicionados por las experiencias, conocimientos y motivaciones de los sujetos (Wolf, 1994). La persuasión es un proceso dependiente de condiciones como el interés del destinatario, la exposición a varias fuentes informativas, las características del emisor y receptor, la cantidad y calidad de mensajes.

En ese sentido, la educación informal en Twitter no es un proceso ligado unidireccionalmente a los medios, sino que se construye como una aceptación colectiva de la comunidad de usuarios que interpreta y socializa el tema en cuestión. De forma que, de acuerdo con la plataforma utilizada, los medios generan información y los receptores se mantienen activos en el proceso.

En Twitter podemos hablar de que la educación informal ligada a la obtención de influencia social se produce gracias a tres momentos en el discurso de los medios:

- Locutivos: enunciación, publicación simbólica del mensaje, como unidad semántica estructurada en 140 caracteres (tuit).

- Ilocutivos: el medio de comunicación, según sus intereses editoriales, construye el tuit con una intencionalidad.

- Perlocutivos: esos tuits periodísticos provocan un efecto en el receptor (retuit, respuesta, favorito).

La educación informal de los públicos respecto a temas mediáticos se produce, primeramente, desde una concepción cognitiva que inicia cuando se establece la agenda mediática.

\section{Agenda setting o disposición temática}

La teoría de la agenda setting, o construcción de la agenda mediática, dispone qué temas informativos dan una mayor importancia e influyen en la agenda del público. En un artículo de 2005, Maxwell McCombs hace una revisión de la teoría de la agenda setting y su aplicación a espacios de la web. En este empeño tiene en cuenta tres elementos: 
1) Concerniente a la división del espacio digital. Gradualmente esta división está siendo superada por una disminución de los precios de las computadoras y más ofertas de instalaciones de acceso público a la web.

2) Relacionada con los hábitos de comunicación de la audiencia en los sitios de la web.

3) La suposición de que la audiencia de internet se dispersa ampliamente a través de diversos sitios en la Web, una situación que podría revelar la desaparición de la teoría de la agenda setting como la conocemos. (pp. 545-546)

Estas conclusiones nos ponen al corriente de una hipótesis que defiende: que las agendas a las que los usuarios se exponen en la Web son divergentes al establecimiento de agenda en los nuevos medios tradicionales; sin embargo, la investigación en torno al tema debería controlar cómo los nuevos medios están representando funciones sociales.

En Twitter los usuarios construyen esquemas de seguimiento de la agenda mediática a través de etiquetas, denominadas hashtags, de modo que aunque el establecimiento de la agenda setting sea divergente con respecto a los medios tradicionales, se pueden seguir los patrones de interpretación de los acontecimientos noticiosos a través de esta estructura temática que nos ofrece un referente contextual.

Los enlaces sociales entre los usuarios de internet estarían determinando la producción de sentido en torno a las agendas de comunicación y, de igual forma, cómo estos enlaces sociales agrupados en comunidades y su concerniente significación influyen en la agenda mediática a través de la agenda pública.

Los medios de comunicación aparecen como instituciones que median entre el acto interpretativo concreto y las prácticas discursivas de una comunidad dada, en un flujo interactivo recíproco en el que intervienen múltiples niveles de producción y circulación de sentido (Redondo, 2009). En este sentido, es importante reconocer que los medios favorecen la referencia cognitiva respecto al hecho noticioso. Cuando ofrecen la noticia proponen la agenda mediática, aunque la organización textual de dicha agenda es un proceso negociado, dado que el hashtag, como estructura semántica que permite seguir el establecimiento de agenda, puede ser habilitado por los medios o estructurado por otro usuario.

\section{Influencia social en Twitter}

La influencia social en Twitter es la potencialidad de la acción de un usuario (por ejemplo: to tweet -tuitear-) de iniciar futuras acciones (por ejemplo: retuit o respuesta) de otros en la red, lo cual se evidencia en la acción de crear un mensaje (tuit) y suscitar su posterior re-difusión (retweet -retuit-) por su red de contactos y de las redes de quienes lo reciben y deciden reenviarlo (Leavitt, Burchard, Fisher y Gilbert, 2009). En Twitter persisten dos tipos de influencia:

1. La basada en la conversación: potencialidad del usuario para influir $y$, por tanto, incitar a otros a iniciar o participar en una conversación. Esta acción se manifiesta mediante la respuesta. El propósito de la respuesta indica que una conversación es el objetivo intencional de la acción. Al escribirla, el usuario ha sido influenciado a responder a una unidad previa de contenido (tuit) publicado por otro en la plataforma.

2. La basada en el contenido: potencialidad del contenido del mensaje de un usuario para influir y, por tanto, lograr que otro(s) lo reproduzca $(n)$, total o parcialmente, para sus seguidores. Dicha influencia se percibe en la acción de retuit, cuyo objetivo principal es difundir contenido.

De igual forma la categorización de tuit como favorito, aunque no es tratada por Leavitt, Burchard, Fisher y Gilbert (2009), es una forma de influencia social basada en el contenido. Se trata de que al seleccionar un tuit como favorito estamos ante un proceso de asimilación y aceptación por parte del usuario.

La influencia social en Twitter se fundamenta en hacer llegar el mensaje al mayor número posible de usuarios, sean o no seguidores de quien los emite. El 
proceso de recepción del mensaje está mediado por el hecho de que, según An, Cha, Gummandi y Crowcroft (2011), el $80 \%$ de los usuarios en Twitter siguen cerca de 10 medios de comunicación, aproximadamente, y tienen contacto con otros 27 vía retuit.

Wu, Hofman, Mason y Watts (2011) constataron que el $46 \%$ de los mensajes de los mass media alcanza a los tuiteros a través de intermediarios, usuarios que reciben la información directamente de los medios y que la replican mediante retuits. Los usuarios son los que seleccionan la información, la filtran, la distribuyen y aumentan o no su alcance a través de enlaces sociales.

Por lo general, los medios de comunicación tienen mayor éxito en la obtención de influencia mediante el retuit, que a través de las opciones de respuesta (Leavitt, Burchard, Fisher y Gilbert, 2009). En Twitter se produce un segundo nivel de filtración de noticias, por cuanto se produce una influencia guiada por usuarios de referencia que, siguiendo la explicación de Canavilhas (2011), "redistribuyen la información a una comunidad de seguidores" sin necesidad de que estos estén vinculados directamente al mensaje publicado por el medio. Entonces, la influencia de los medios de comunicación depende de las reacciones que suscita, tanto por repetición y asimilación, como por respuesta sobre los miembros de la comunidad virtual que sigue la noticia.

\section{El usuario y su agrupación en comunida- des virtuales}

El usuario de internet es aquel que utiliza un servicio web, ya sea en función de visualizarlo, administrarlo, editar, colaborar o suscribirse. Según Nafría (2007), es el rey de la Web actual en su versión 2.0, ya que pasa de ser mero espectador y consumidor de lo que se le ofrece en internet a convertirse en creador y generador de contenidos y servicios.

Las condiciones del usuario varían dadas las posibilidades que le ofrecen los sitios en la web. Así, por ejemplo, en las redes sociales los usuarios no solo son editores de sus cuentas y productores de contenidos, también se agrupan en comunidades virtuales que favorecen la creación de grupos enlazados socialmente por intereses comunes.

El término de comunidades virtuales es usado para referirse a un grupo de personas que se asocian entre ellas por un tiempo en un ambiente mediado por computador, con énfasis en los comportamientos sociales del grupo. Según Herring (2008):

Las comunidades virtuales en internet como grupos sociales en si pueden ser agrupadas en cinco clases: grupos de interés (ejemplo fans a la telenovela), grupos de apoyo (por ejemplo, relacionados con la salud), grupos basados en cercanía geográfica y ambientes comerciales. (Herring, 2008, p. 2)

Usualmente los usuarios interesados en acontecimientos noticiosos responden a la categoría grupos de interés. En estos últimos se produce una relación transcultural en la que el propio texto es el medio para la relación de los usuarios. El contexto social de la relación entre los usuarios determina que los miembros de la comunidad virtual:

Compartan metas, intereses, necesidades, o actividades que proporcionan las razones principales para pertenecer a las comunidades.

Repitan acciones como participación activa, a menudo tienen interacciones intensas, fuertes relaciones emocionales y actividades compartidas entre participantes.

Tengan acceso a compartir recursos y existan políticas para determinar el acceso a estos recursos.

Haya un contexto compartido de convenciones sociales, lenguaje y protocolo. (Preece y Maloney-Krichmar, 2003, p. 597)

En este sentido, Watts (2006) menciona que la importancia de las interconexiones entre estos sujetos "no radica únicamente en la identificación de las características o número de elementos que conforman algún sistema, sino en las relaciones, interdependencias e interacciones que se dan entre ellos" (p. 62). El autor determina que las relaciones en redes virtuales siguen un orden; cualquier red puede ser una red de mundo pequeño con tal de que tenga algún modo 
de plasmar el orden y continúe re-teniendo alguna pequeña cantidad de desorden. El origen del orden puede ser social, esa conexión entre nodos tiene a la sociología como fuente de estímulo. A partir de los enlaces sociales y la distribución de contenidos se genera una comunidad interpretativa ${ }^{2}$.

\section{Metodología}

Partiendo de criterios expuestos en los referentes conceptuales, se formuló la siguiente idea a defender:

En Twitter los medios de comunicación influyen socialmente en los usuarios a través de la enunciación informativa en la disposición de la organización textual, la distribución de sus mensajes mediante enlaces sociales por patrones de repetición-reacción, y la creación de consenso sobre la interpretación de acontecimientos por usuarios miembros de la comunidad.

El objeto de investigación fueron los tuits asociados a la VII Cumbre de la AEC emitidos por los medios institucionales y por usuarios bajo las etiquetas (hashtags) \#CubaCaribe, \#VIICumbreAEC, \#CumbreAEC.

En tal sentido fue pertinente analizar las interacciones comunicativas entre usuarios y medios de comunicación, así como el proceso de interpretación y valoración del acontecimiento noticioso en la comunidad virtual, tomando al análisis del discurso mediado por computador como perspectiva metodológica.

\section{Análisis del discurso mediado por computador}

Según Sampieri et al. (2014, p. 420), el análisis del discurso como acercamiento propone una revisión temática que proporciona herramientas analíticas que permiten efectuar continuas reflexiones durante la inmersión profunda en el campo sobre los datos recolectados y las impresiones respecto al ambiente.

2 Una comunidad interpretativa es un grupo de interpretantes que generan una significación sobre un referente dado. Charles Sanders Peirce propone que, para legitimar la verdad o la realidad de algo, es imprescindible que un cierto número de personas (intérpretes) con unas mismas características, se pongan de acuerdo en que ese algo sea real. Este grupo social concreto es denominado por Jensen (1997) como comunidad interpretativa.
La presente investigación utilizó como método el análisis del discurso mediado por computador para determinar cómo se produce la influencia de los medios de comunicación sobre los usuarios en la interpretación de la VII Cumbre de la Asociación de Estados del Caribe.

La comunicación mediada por computadores se entiende como un conjunto de modalidades de interacción que emergen a partir de la aplicación de las tecnologías de investigación y comunicación a la comunicación pública e interpersonal. El análisis del discurso mediado por computador propone un acercamiento a la comunicación en escenarios reticulares desde la metodología del análisis del discurso. Su principal objetivo es, de acuerdo con Mancera y Pano (2013), analizar las propiedades lingüísticas y pragmáticas de este tipo de interacciones, a través de la descripción y clasificación de las formas discursivas que surgen en los entornos virtuales.

Esta perspectiva metodológica (propuesta por Herring, 2002) envuelve análisis cualitativo y cuantitativo; pero lo que lo define en su totalidad es el estudio de registros de interacción verbal. Para ello propone el abordaje de estructura, significado, interacción, comportamiento social y participación en el contexto de comunidades de interpretación.

La aplicación del análisis del discurso mediado por computadora es pertinente cuando se realizan investigaciones sobre las nuevas dinámicas conversacionales surgidas en el escenario de la Web 2.0. Mancera y Pano (2013) aseguran que en Twitter se generan nuevas dinámicas discursivas a través de dispositivos como el retuit y las etiquetas (o hashtags), que reconfiguran en el discurso los procedimientos de cita, la coherencia textual, la gestión de la alternancia de turnos o la intertextualidad.

En el análisis de la influencia de los medios de comunicación en Twitter el análisis del discurso mediado por computador supone un análisis textual que permite reconocer condiciones objetivas y subjetivas de producción, circulación y consumo de los mensajes mediáticos. Y, de igual forma, facilita representar los rasgos de una relación discursiva para descubrir órdenes, reglas y regularidades en el discurso, y 
reconocer los modos en los que los medios de comunicación influyen sobre el público.

En este sentido, con el estudio del discurso se logra un grado de interpretación más profundo del recorrido textual, para determinar los procesos por los que se da la influencia de los medios de comunicación en la reproducción por medio del lenguaje o de los grupos sociales de determinado mensaje o ideología. Así, el discurso constituye el lugar donde los medios de comunicación y los sujetos construyen la interpretación en un proceso de negociación supeditado al contexto particular del microblogging, según acciones propias de la interfaz ${ }^{3}$ de la plataforma.

Ahora bien, atendiendo a la interfaz, se debe tener en cuenta que el contexto discursivo se caracteriza por:

- Organización textual común (hashtag).

- Los participantes comparten sus percepciones, dialogan entre ellos y participan en la interpretación sobre el acontecimiento (tuit, retuit, respuesta, favorito).

- Los usuarios pueden haber estado expuestos a otras fuentes comunicativas de naturaleza virtual o analógica.

Tomando como unidad de análisis el tuit, el análisis del discurso mediado por computador se realizó a partir de la siguiente guía:

1. Análisis de estructura.

1.1 Selección de la muestra. Organización textual que engloba el acontecimiento.

1.2 Análisis de los participantes del discurso (medios de comunicación - otros usuarios).

2. Análisis de patrones de repetición y reacción (aplicado a los tuits publicados por medios de comunicación).

- Influencia social basada en el contenido (retuit - favorito).

3 La interfaz determina las posibilidades que da la plataforma para editar el contenido. Por ejemplo, la de Twitter permite mecanismos de interacción como el retuit, la respuesta, la publicación de un tuit, mención, selección de favoritos.
- Influencia social basada en la conversación (respuesta).

- Usuarios influyentes que citaron a través de retuits los mensajes de las cuentas de instituciones mediáticas.

3. Análisis fino (postura discursiva de los medios de comunicación).

4. Análisis global (postura discursiva de la comunidad virtual respecto a la postura discursiva de los medios de comunicación - cambios en la dirección de la conversación).

En el análisis global se aplicó el siguiente esquema de evaluación para determinar la interpretación del acontecimiento por el conjunto de usuarios de la comunidad virtual:

Actitud (tipo: afecto, juicio, apreciación).

Expresión (directa o evocativa).

Polaridad (positiva o negativa).

Compromiso (personal-grupal).

Gradual (apoyo-enfoque).

\section{Corpus de estudio}

El análisis del discurso mediado por computador fue aplicado a un corpus de 1500 tuits que fueron sustraídos del microblogging a partir de la aplicación Follow the Hashtag 4 , la cual proporciona un informe completo de la influencia social de los usuarios, así como la sustracción del contenido de los tuits.

El análisis partió de la selección de un conjunto de textos que responden a enunciaciones sobre la VII Cumbre de Asociación de Estados del Caribe. Para ello se seleccionaron los tuits con las etiquetas temáticas \#CubaCaribe, \#VIICumbreAEC, \#CumbreAEC que guían la conversación sobre el acontecimiento noticioso. Téngase en cuenta además que, dada la perspectiva metodológica utilizada y la muestra en cuestión, se evaluó a los usuarios activos, es decir a aquellos que generaron y transmitieron contenido dentro del grupo.

4 www.followthehashtag.com 


\section{Análisis de los resultados}

\section{Análisis de estructura}

\subsection{Selección de la muestra. Organización textual que engloba el acontecimiento}

Muestra : 1500 tuits.

Organización textual que engloba el acontecimiento: \#CubaCaribe, \#VIICumbreAEC, \#CumbreAEC.

\subsection{Análisis de los participantes del discurso (medios de comunicación - otros usuarios)}

Participantes de la comunicación:

716 usuarios: 14 medios de comunicación institucionales/702 otros usuarios.

Cuentas en Twitter de medios de comunicación institucionales que intervienen en la conversación: @trabajadorescu, @Juventud_Rebelde, @Granma_ Digital, @EmisoraGuaimaro, @radionline, @enciclopedia_cu, @RadioTainoFM, @tvcamaguey, @ cubadebate, @SputnikMundo, @notiminuto_vzla, @ teleSur_Cuba, @radioflorida, @PrensaLatina_cu.

Los primeros tuits asociados a las etiquetas seleccionadas fueron publicados en la mañana del 2 de junio de 2016 por las cuentas @Granma_Digital, @Prensa Latina_cu y @cubadebate, lo que denota que el inicio de la conversación sobre el tema fue iniciado por cuentas institucionales de medios de comunicación.

\section{Patrones de repetición}

Los mensajes de los medios de comunicación son distribuidos mayoritariamente a través de patrones de repetición basados en la asimilación del tuit, mediante el retuit. Por tanto, se produce un grado de acumulación y en menor medida los parlamentos mediáticos suscitan reacciones basadas en la respuesta del público.

2.1 Usuarios influyentes que citaron a través de retuits los mensajes de las cuentas de instituciones mediáticas

La distribución de los tweets de medios de comunicación es realizada por usuarios influyentes, a través de conexiones sociales. Los usuarios influyentes que citaron a través de retuits los mensajes de las cuentas de instituciones mediáticas fueron: @SalvadorHolguín, @adrielfcuban, @YusmilaReyna, @Guajiritasoy, @DiegoPerez1204, @norellysmorales, @ AnaTeresitaGF, @vencancilleria. Estos reprodujeron el contenido publicado y facilitaron su distribución a sus seguidores y a la comunidad.

\section{Análisis fino (postura discursiva de los medios de comunicación)}

En el caso de estudio presentado y a los efectos del análisis del discurso mediado por computador, podemos reconocer que los 14 medios que reportan información sobre la VII Cumbre de la Asociación de Estados del Caribe lo hacen desde un perspectiva positiva con respecto al hecho. Algunos argumentos, presentados por las cuentas de los medios de comunicación, que validan esta interpretación son:

- La VII Cumbre de la Asociación de Estados del Caribe contó con una participación de representantes, superior a la de anteriores cumbres de la asociación.

@ cubadebate: Alfonso Múnera: esta Cumbre \#AEC ha sido la de mayor participación de alto nivel. \#CubaCaribe \#Cuba https://t.co/qjqG5OajoM

- El evento fue exitoso, asistentes a la cumbre agradecieron la hospitalidad y organización del mismo por el estado cubano.

@PrensaLatina_cu: \#CumbreAEC exitosa, debate sustancioso, prevalecieron la unidad, armonía y coincidencia en los temas fundamentales. http... 


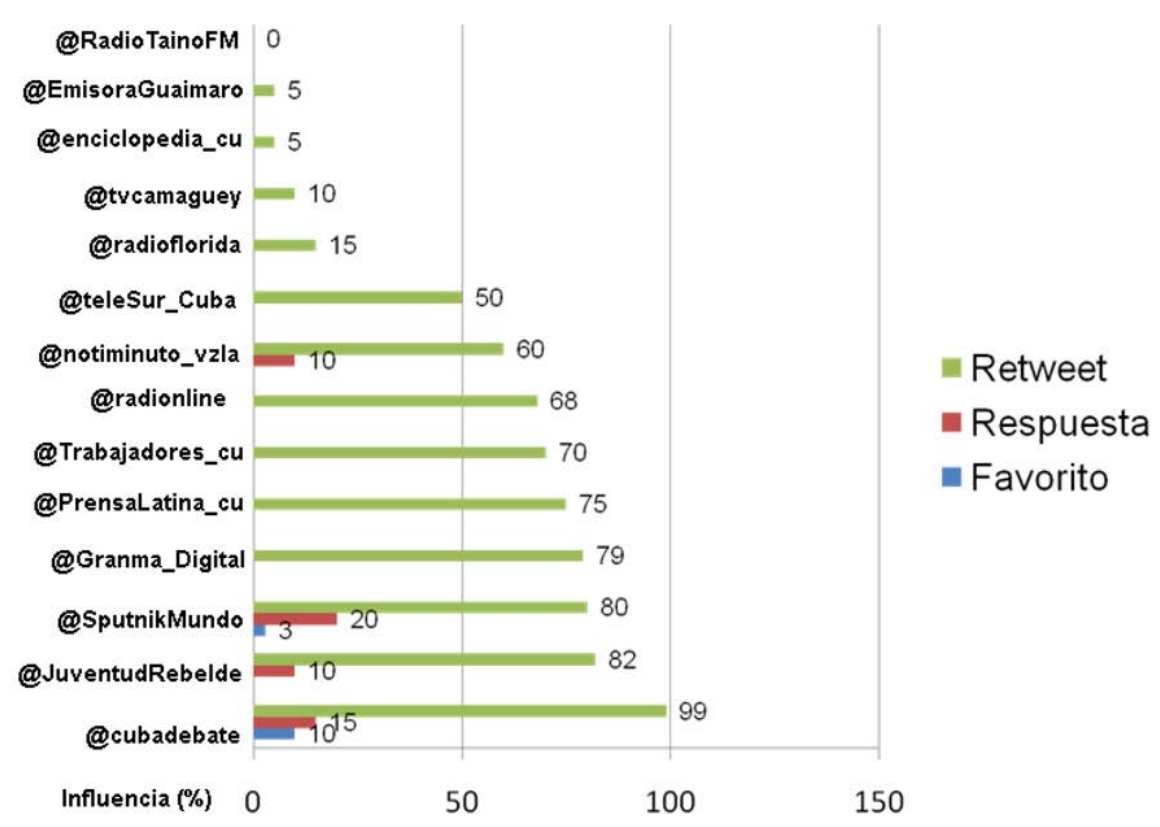

Figura 1. Influencia social de los mensajes de los medios de comunicación

Fuente: elaboración propia.

@Trabajadores_cu: Raúl Castro recibió a varios mandatarios tras la VII Cumbre de la \#AEC =\&gt; https://t.co/c28ijqDG05 \#Cuba \#CubaCaribe htt...

@enciclopedia_cu: \#Cuba Alfonso Múnera: este país me ha recibido como a un cubano más \#AEC \#CubaCaribe https://t.co/aEQYWs8xQp

- La Asociación de Estados del Caribe se consolida como organismo de consulta y defensa de los Estados de la región.

@ radioflorida: Raúl Castro \#Cuba \#AEC se confirma como organismo de consulta, concertación y cooperación en el Caribe. \#CubaCaribe

- Los temas más tratados asociados a la Cumbre en el discurso de los tuits de la muestra fueron: cambio climático, integración, injerencia extranjera en los Estados del Caribe y su soberanía, el destino de los pueblos del Caribe, mejoras en la calidad de vida, amenazas a la paz y seguridad nacional en la región.

@radioonline: Curazao aboga por una mayor calidad de vida para los más vulnerables a los más débiles \#VIICumbreAEC \#CubaCaribe
@Trabajadores_cu: Raúl Castro \#Cuba VII Cumbre \#AEC hay q enfrentar amenazas a la paz y la seguridad internacionales. \#CubaCaribe https...

@cubadebate: @NicolásMaduro estamos viviendo lo que adviritó @ reflexionfidel en tiempos de \#CambioClimático \#CubaCaribe https://t.co/JQP7 ...

@PrensaLatina_cu: Cumbre caribeña defiende paz, integración y desarrollo sostenible \#AEC \#CubaCaribe https://t.co/yclOdKWLwB

\section{Análisis global (postura discursiva de la comunidad virtual respecto a la postura discursiva de los medios de comunicación - cambios en la dirección de la conversación}

Se evaluó el discurso de la comunidad virtual respecto al hecho noticioso, en este sentido las valoraciones de los usuarios coincidieron, en su mayoría, con las que hacen los medios de comunicación sobre la VII Cumbre de Asociación de Estados del Caribe. La interpretación, polaridad y juicio sobre el suceso es mayoritariamente positiva. 


\section{Cambios en la dirección de la conversación}

No obstante, en la muestra se observan ejercicios valorativos de usuarios con una posición negativa respecto a la cumbre, sobre todo en la inserción de temas que constituyen cambios en la dirección de la conversación. El discurso de las cuentas @arlencezar (por palabras clave repetidas), @omarbula (por influencia social al $67 \%$ a través de retuits) se insertó en la comunidad virtual relacionando la Cumbre con los esfuerzos de \#UNPACU, la mafia internacional y a Cuba con los Papeles de Panamá. Dichos parlamentos fueron una forma de contrainformación al discurso de los medios cubanos y otros usuarios con una percepción positiva del evento.

@ arlencezar: \#FelicidadesRaul \#FidelCastro \#Cuba \#CubaCaribe \#cubaSenCastros \#SocialismoCubano \#UNPACU \#TodosMarchamos q https://t.co/ npAljoCDTk

@arlencezar: Cuba en los Papeles de Panamá - Patria de Martí https://t.co/ZQMez9BYOR via @ JulioMShiling \#SocialismoCubano \#CubaCaribe \#TodosMarchamos @arlencezar: \#CubaCaribe \#TodosMarchamos \#UNPACU ABAIXO DITADURA, ABAIXO FIDEL \#CubaSenCastros \#RaulCastro \#Cuba t https://t.co/ csSrFUmowg

@omarbula: Mafia internacional de la DROGA \&amp; CRIMEN respalda el \#ProcesoDePaz \#CumbreAEC @geopolytica https://t.co/dnr3Fv3hXa

\section{Discusión de los resultados}

Partiendo del caso de estudio, de su interpretación, de los enlaces sociales y de la distribución del mensaje, podemos afirmar que la influencia social de los medios de comunicación en Twitter sobre los usuarios en el caso de la VII Cumbre de la Asociación de Estados del Caribe se produce de la siguiente forma:

1. Enunciación temática (los medios de comunicación tienen éxito en comenzar la conversación dentro de la comunidad virtual). Las cuentas institucionales de los medios de comunicación fueron los primeras en publicar y

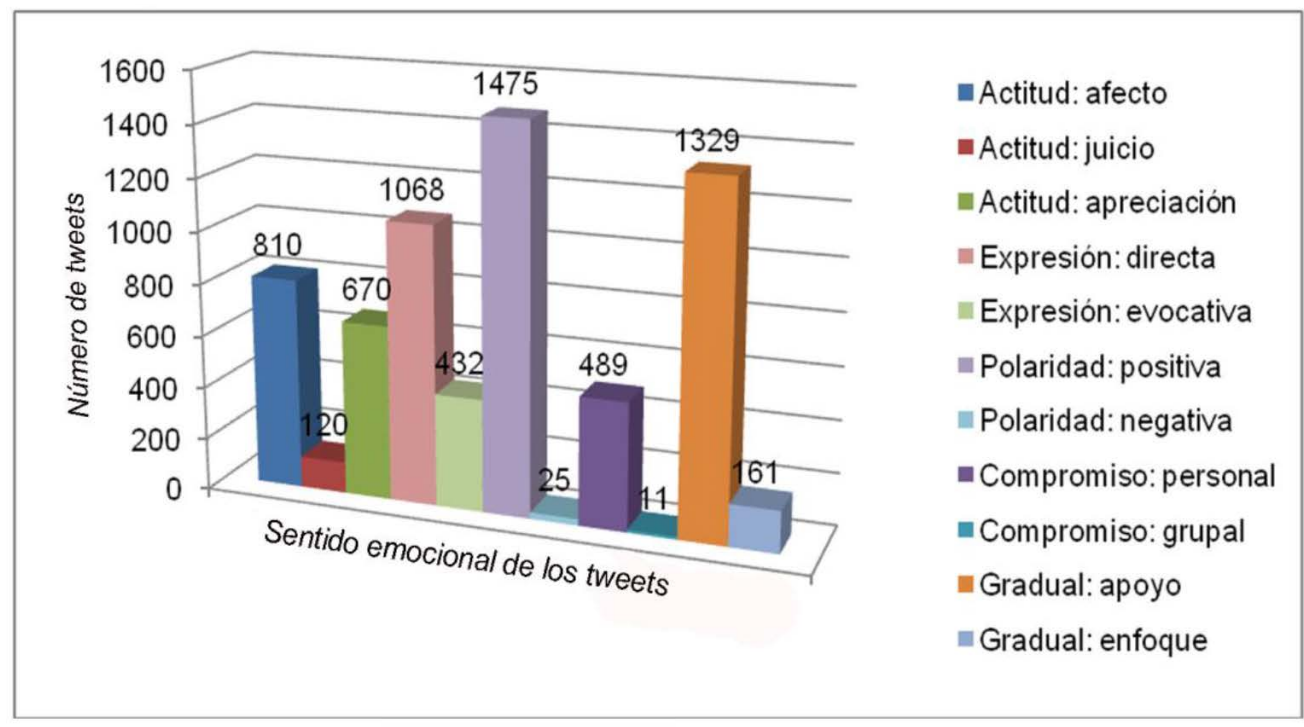

Figura 2. Evaluación del discurso por número de tweets de la muestra

Fuente: elaboración propia. 
utilizar las etiquetas \#CubaCaribe, \#VIICumbreAEC, \#CumbreAEC para comenzar la conversación sobre la VII Cumbre de la Asociación de Estados del Caribe. Esto supone que los medios expresaron la agenda mediática para luego ser comentada por otros usuarios.

2. Reproducción de los mensajes mediáticos.La influencia de los medios en Twitter, con respecto a la VII Cumbre de la Asociación de Estados del Caribe, se produce mayoritamente por asimilación-reproducción a través del retuit de los mensajes publicados por las cuentas institucionales de entidades mediáticas. En menor medida, las cuentas institucionales de los medios de comunicación logran suscitar una reacción a través de la respuesta.

3. Distribución a través de enlaces sociales. Los tuits de los medios alcanzan una mayor audiencia gracias a la reproducción a través del retuit por parte de usuarios influyentes quienes redistribuyen el contenido y permiten que el contenido sea expuesto a otros usuarios de la comunidad virtual.

4. Consenso en la percepción-interpretación.La interpretación del evento fue evaluada como positiva por la mayoría de los participantes en la conversación, en correspondencia con las valoraciones que dan los medios sobre el evento. No obstante, se produjeron cambios en la dirección de la conversación, los cuales dieron lugar a otras interpretaciones, aunque menos influyentes en la comunidad con respecto al consenso ya logrado por el grupo.

\section{Conclusiones}

La influencia social de los medios de comunicación en Twitter, durante la VII Cumbre de Estados del Caribe, se produjo como una forma de educación informal que ofreció los elementos conceptuales, cognitivos e interpretativos para la interpretación del acontecimiento por el grupo de usuarios de la comunidad virtual. Agrupados bajo las etiquetas \#CubaCaribe, \#VIICumbreAEC, \#CumbreAEC, los participantes de la conversación dispusieron la organización textual de la agenda mediática.

Para efectos de la presente investigación, en Twitter los medios de comunicación influyen socialmente en los usuarios a partir de elementos como la enunciación informativa, la reproducción de los mensajes a través de patrones de repetición como los retuits o la distribución de su contenido a través de usuarios influyentes dentro del grupo. De la misma manera, tienen particular éxito en construir el consenso respecto a la interpretación como forma de aceptación colectiva de los parlamentos mediáticos.

\section{Referencias bibliográficas}

An, J.; Cha, M.; Gummandi, K. y Crowcroft, J. (2011). Media Landscape in Twitter: A World of New Conventions and Political Diversity. Recuperado de http://www.cl. cam.ac.uk/ jac22/out/twitter-diverse.pdf.

Bergman, M. (2000). Meaning and Mediation: Toward a Communicative Interpretation of Peirce's Theory of Signs. Helsinki: Department of Communication, University of Helsinki.

Buckingham, D. (2005). Educación en medios. Alfabetización, aprendizaje, y cultura contemporánea. Barcelona: Paidós.

Burns, A. (2003). Gatewatching, not Gatekeeping: Collaborative Online News. Media International Australia Incorporating Culture and Policy: Quarterly Journal of Media Research and Resources, 107, 31-44. Recuperado de http://eprints.qut.edu.au/189/

Burns, A. y Highfield, T. (2015). From News Blogs to News on Twitter: Gatewatching and Collaborative News Curation. En S. Coleman y D. Freelon (eds.), Handbook of Digital Politics (pp. 325-339). Nueva York: Routledge.

Canavilhas, J. (2011). Del gatekeeping al gatewatching: el papel de las redes sociales en el ecosistema mediático. En F. Irigaray, D. Ceballos y M. Manna, Periodismo digital: convergencia, redes y móviles (p. 154). Rosario: Universidad Nacional de Rosario; Fundación La Capital. Recuperado de http://www.fpdrosario.com.ar/foro/2011/06/ periodismo-digital-convergencia-redes-y-moviles. 
Gruzd, A.; Wellman, B. y Takhteyev, Y. (2011). Imagining Twitter as an Imagined Community. American Behavioral Scientist, 55(10), 1294-1318. Recuperado de http://groups.chass.utoronto.ca/netlab/wp-content/ uploads/2012/05/Imagining-Twitter-as-an-Imagined-Community.pdf

Hernández S., R.; Fernández C., C. y Baptista L., P. (2014). Metodología de la investigación. 6a. ed. Ciudad de México: Editorial McGraw-Hill.

Herring, S. C. (2002). Computer-mediated Communication on the Internet. Annual Review of Information Science and Technology, 36(1), 109-168. Recuperado de sites. google.com/site/dozwang/fulltext3.pdf

Herring, S. C. (2008). Virtual Community. En L. M: Given (ed.), Encyclopedia of Qualitative Research Methods (s. p). Nueva York : Sage. In press.

Jensen, K. B. (1997). Semiótica social de la comunicación de masas. Barcelona: Editorial Bosch.

Leavitt, A.; Burchard, E.; Fisher, D. y Gilbert, S. (2009). The Influentials: New Approaches for Analyzing Influence on Twitter. Recuperado de: http://www.webecologyproject.org/wp-content/uploads/2009/09/ influence-report-final.pdf

Liceras, A. (2005). Los medios de comunicación de masas, educación informal y aprendizajes sociales. Didáctica de las Ciencias Sociales, Geografía e Historia, 46, 109-124.

Loo, J. R.; Gámez, D.; Lamarque, E.; Domínguez, L. y Haber, Y. (2015). Del gatekeeper al content curator: cambiar algo para que no cambie nada. Revista Razón y Palabra, 19(92), 1-23. Recuperado de http://www.razonypalabra.org.mx/N/N92/Varia/21_LooGamezLamarqueDominguezHaber_V92.pdf

Mancera, A. y Pano, A. (2013). El discurso político en Twitter. Madrid: Editorial Anthropos.
McCombs, M. (2005). A Look at Agenda Setting: Past, Present and Future. Journalism Sudies, 6(4), 543-557.

Nafría, I. (2007). Web 2.0: el usuario, el nuevo rey de Internet. Barcelona: Gestión 2000.

Preece, J. y Maloney-Krichmar, D. (2003). Online Communities. En J. Jacko y A. Sears (eds.), Handbook of Human-Computer Interaction. Mahwah Lawrence Erlbaum Associates Inc. Publishers.

Redondo, I. (2009). El signo como medio: Claves del pensamiento de C.S. Pierce para una teoría constitutiva de la comunicación (tesis doctoral). Pamplona: Universidad de Navarra, España. Recuperado de http://www. unav.es/gep/TesisDoctorales/TrabajolnvestigacionRedondo.pdf.

Rost, A. (2012). Modelos de uso y gestión de las redes sociales en el periodismo. Ponencia presentada en el IV Congreso Internacional de Ciberperiodismo y Web 2.0, Andoibarra, España.

Searle, J. R. (1997). Actos de habla. Barcelona: Paidós.

Watts, D. (2006). Seis grados de separación. La ciencia de las redes en la era del acceso. Barcelona: Paidós.

Wodak, R. y Meyer, M. (2003). Método de análisis crítico del discurso. Barcelona: Gedisa.

Wolf, M. (1994). Los efectos sociales de los media. Barcelona: Paidós.

Wu, S.; Hofman, J.; Mason, W. y Watts, D. (2011). Who Says What to Whom on Twitter. En 20th International Conference on World Wide Web (pp. 705-714). Recuperado de http://www.research.yahoo.net/files/w_h_m_w_WWW_11.pdf.

Zappavigna, M. (2011). Ambient affiliation: A linguistic Perspective on Twitter. New Media \& Society, 13(5), 788-806. Recuperado de http://nms.sagepub.com/ content/13/5/788 